\title{
Revisiones
}

\section{Plan de retorno al trabajo tras baja laboral por motivos de salud mental}

\section{Plan of return to the work after mental illness and labor absence}

\section{Carmen Otero Dorrego ', Macarena Gálvez Herrer ${ }^{2}$}

1. Médico Especialista en Medicina del Trabajo. Técnico Superior en P.R.L. Auditora en gestión de PRL. Madrid. España.

2. Doctora en Psicología Clínica y de la Salud. Técnico Superior en P.R.L. Madrid. España.

Recibido: 08-04-14

Aceptado: 12-05-14

\section{Correspondencia}

Dra. Carmen Otero Dorrego

Servicio de Prevención de Riesgos Laborales. Hospital Universitario de Móstoles

C/Río Júcar, s/n

28935 Móstoles. Madrid. España

carmen.otero@salud.madrid.org

Resumen

Los autores, siguiendo las recomendaciones internacionales, revisamos la situación actual en nuestro medio y proponemos unas líneas generales de actuación, para asegurar una adecuada reincorporación laboral de la persona, la adaptación del puesto de trabajo (desempeño de tareas, interacción del trabajador con equipos y lugares de trabajo, etc.) y el correcto funcionamiento de la organización. Con ello se pretende evitar situaciones de estigmatización y favorecer la adaptación del trabajo a la persona.

Med Segur Trab (Internet) 2014; 60 (235) 392-405

Palabras clave: salud mental, retorno al trabajo, intervención en el lugar de trabajo, Medicina del trabajo.

\begin{abstract}
The authors, following the international recommendations, we check the current situation in our way and propose a few general lines of performance, to assure a suitable labour reincorporation of the person, the adjustment of the working place (performance of tasks, interaction of the worker with equipments and places of work, etc.) and the correct functioning of the organization. With it one tries to avoid situations of estigmatización and to favor the adjustment of the work to the person.
\end{abstract}

Med Segur Trab (Internet) 2014; 60 (235) 392-405

Key words: Mental health, return to the work, intervention in the workplace, Occupational Health. 


\section{INTRODUCCIÓN}

Los problemas de salud mental, independientemente de su desencadenante, pueden afectar a trabajadores de cualquier sector de producción, asociándose, en muchas ocasiones, a periodos de incapacidad laboral transitorios (IT), o bajas laborales, de larga duración. En ocasiones, la capacidad para realizar el trabajo habitual solo se recupera parcialmente, o no se recupera, dando lugar a incapacidad permanente (IP) en distintos grados. Aunque la recuperación sea completa, la reincorporación al puesto de trabajo tras una ausencia prolongada, puede precisar de medidas adaptativas transitorias.

Cuando se produce la reincorporación laboral, tras una baja de larga duración por motivos de salud mental (art. 37.3.a del Reglamento de los Servicios de Prevención de Riesgos laborales, RSPRL) ${ }^{(1)}$, la adaptación laboral siempre supone un reto para el propio trabajador y para la organización, no sólo por el propio proceso de adaptación de un trabajador que ha permanecido fuera de la organización durante un periodo largo de tiempo recuperándose de una enfermedad, sino también por la propia estigmatización social (prejuicios hacia todos los enfermos con problemas de salud mental, por desconocimiento y miedo a la enfermedad).

Para emitir aptitud y propuestas de adaptación, el médico del trabajo del Servicio de Prevención de Riesgos Laborales (SPRL) deberá contemplar la situación personal del propio trabajador al alta, las condiciones de su puesto de trabajo y las características de la organización. Se contemplarán adaptaciones, entre otras, en situaciones especiales (turno nocturno, conducción de vehículos, tareas de elevada responsabilidad en cuanto a riesgo a terceras personas...), asegurando una reincorporación laboral segura para el propio trabajador [Trabajador Especialmente Sensible (TES) según el art. 25 de la Ley de Prevención de Riesgos laborales (LPRL) ${ }^{(2)}$ frente al riesgo organizativo de estrés] y para su entorno laboral (riesgo a terceros: compañeros de trabajo, usuarios de su tarea o servicio, organización).

La coordinación integral e integrada de recursos laborales dentro de un Plan de Ayuda al Empleado ${ }^{(3)}$ (Employee Assistance Programmel, EAP), por una parte, así como de recursos sanitarios (coordinación efectiva entre el médico del trabajo del SPRL y los posibles recursos de la Red Sanitaria Pública, para evitar altas laborales precoces o inadecuadas) es fundamental para conseguir un adecuada reincorporación laboral. Un ejemplo práctico, siguiendo recomendaciones internacionales (proyecto europeo Good Work, Good Health ${ }^{(4)}(\mathrm{GWGH}, 2010)$, European Survey of New and Emerging Risks o Estudio ESENER ${ }^{(5)}$, OSHA-2012, entre otros), consiste en la elaboración de guías de buenas prácticas, como The Good Practice Guidelines on how to improve the mental well-being of workers in the telecommunications sector $(2010)^{(6)}$, desarrollada dentro del marco de diálogo social europeo y patrocinada por la Comisión Europea y el Institute of Occupational Medicine de Edimburgo (IOM).

\section{Los trastornos de salud mental en el trabajo: ¿un problema de diagnóstico o de competencias?}

Según la OMS, la salud mental se define como un estado de bienestar en el cual el individuo es consciente de sus propias capacidades, puede afrontar las tensiones normales de la vida, puede trabajar de forma productiva y fructífera, y es capaz de hacer una contribución a su comunidad.

La pérdida de este estado de bienestar se considera trastorno o enfermedad mental cuando provoca una alteración de tipo emocional, cognitivo y/o del comportamiento, en la que se afectan procesos psicológicos básicos (la emoción, la motivación, la cognición, la conciencia, la conducta, la percepción, la sensación, el aprendizaje, el lenguaje, etc.), dificultando la adaptación de la persona a su entorno (socio-familiar y laboral) y se percibe como malestar subjetivo (Gálvez, Mingote, Núñez y Otero, 2012)(7). Suele ser multifactorial. Se trata de un proceso de interrelación entre factores desencadenantes (estresores laborales y/o extra laborales) y efectos (estrés), que se potencian unos a otros 
hasta que se ocasiona un daño a la salud (alteración de los procesos psicológicos básicos) que deteriora la funcionalidad del individuo, no solo en la esfera laboral, sino también en otros aspectos de su vida, abocando a la necesidad de tratamiento por un profesional de la salud mental (psiquiatra, psicólogo...), y un periodo de baja laboral más o menos prolongado.

Figura 1. Interrelación entre los factores de riesgo de pérdida del estado de bienestar mental

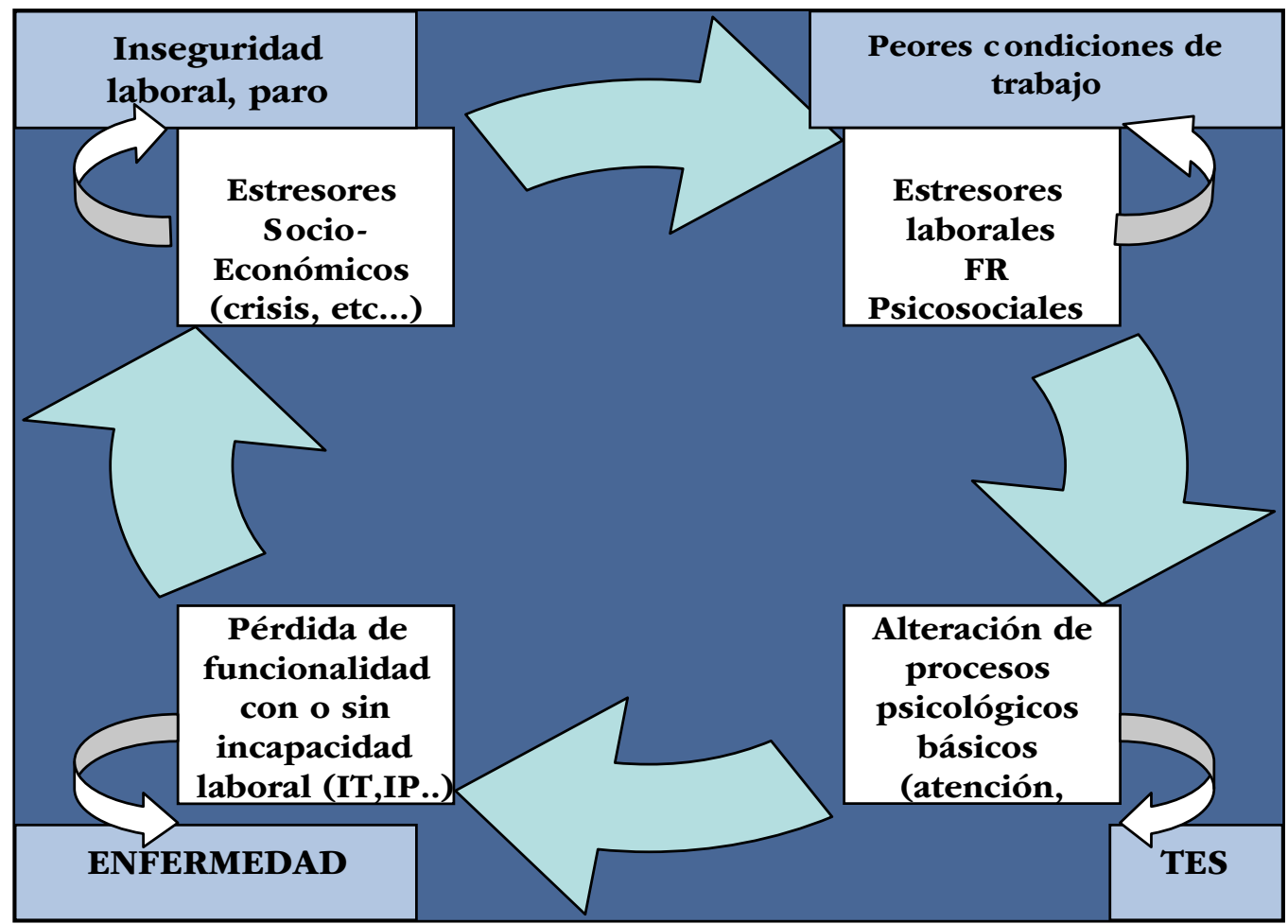

De modo que no sólo el trabajo influye en la salud, sino que también la pérdida del estado de bienestar mental repercute negativamente en el entorno laboral

\section{A) Influencia del trabajo en la salud}

Desde que, en el año 2003, la Agencia Europea para la Seguridad y la Salud en el Trabajo $^{(8)}$ alertase de que el estrés afectaba a más de la cuarta parte de la población trabajadora, se ha evidenciado la necesidad de prevenir los factores de riesgo psicosocial ${ }^{(9)}$. El estudio ESENER corrobora que, en la actualidad, el estrés representa más del 50\% del absentismo en las empresas (estrés, depresión y ansiedad) y que en Europa más de uno de cada cuatro trabajadores sufre estrés ${ }^{(5)}$.

Los factores psicosociales son percepciones que se producen en cada trabajador, resultado de las interacciones que existen entre las condiciones en que desarrolla su trabajo (complejidad de la tarea, medio ambiente de trabajo, satisfacción y condiciones de organización) y su propia capacidad para trabajar (situación personal, cultural y social), influenciadas por las experiencias previas. Pueden percibirse como positivos o como negativos (desfavorables para la calidad de vida del individuo y la actividad laboral).

Se habla de factores de riesgo psicosocial o riesgos psicosociales, cuando los factores psicosociales se perciben como negativos (fuentes de estrés laboral), y tienen el potencial de causar daño psicológico, físico, o social a los individuos. El estrés es una respuesta adaptativa o proceso que puede afectar al estado de salud del propio trabajador (somatizaciones, alteración de la esfera cognitiva o emocional, etc.) y a la seguridad en el trabajo (p.ej., accidentes laborales debidos a un déficit de atención). 
Es previsible que la cifra de trabajadores que sufren las consecuencias del estrés siga aumentando proporcionalmente al empeoramiento de las condiciones socioeconómicas [Gil-Monte $\left.{ }^{(10)}, 2009\right]$. En el estudio ESENER (OSHA, 2012)(5) se contempla el impacto de la crisis en la salud mental de los trabajadores: el objetivo de los empresarios se centraba en aumentar la productividad en un $20 \%$, aumentando el rendimiento de los trabajadores (no el número) y el 55\% de los trabajadores estudiados aseguraban que no se veían capaces de hacer frente durante mucho más tiempo al estrés que les generaba su trabajo.

Según la VII Encuesta de Condiciones de Trabajo en España(11), elaborada por el Instituto Nacional de Seguridad e Higiene en el Trabajo (INSHT, 2011) los indicadores relativos a las exigencias derivadas de factores psicosociales del trabajo (nivel de atención exigida en la tarea, percepción de tener mucho trabajo y sentirse agobiado, tener que trabajar muy rápido o deber atender varias tareas al mismo tiempo) habían empeorado respecto a los reflejados en 2007. Los trabajadores presentaron mayores porcentajes de sintomatología psicosomática. Para la mayoría, sus problemas de salud (trastornos musculoesqueléticos, cansancio, agotamiento, y estrés) estaban relacionados por el trabajo que realizaban.

Para prevenir los efectos de los riesgos psicosociales (Figura 2) se debe realizar una Evaluación de Riesgos Psicosociales (ERP), proceso dirigido a estimar la magnitud de aquellos riesgos que no hayan podido evitarse, obteniendo la información necesaria para adoptar medidas preventivas (control de riesgos) que exige la LPRL, pero no las consecuencias propiamente dichas de los mismos (NTP 702) (12).

Síntomas precoces de estrés, burnout (NTP 704, 705 y 732) (13-15) y mobbing o acoso moral en el trabajo (NTP 854) ${ }^{(16)}$, son consecuencias personales de la exposición a determinados factores de riesgo psicosocial. Se abordan en Planes específicos en las empresas, y su detección precoz se realiza en el ámbito de la vigilancia de la salud individual de los trabajadores expuestos a riesgos de estrés organizativo (Gálvez, Mingote, Núñez y Otero, 2012)(7).

Para aumentar la complejidad del problema, es importante señalar que la percepción del estrés presenta variabilidad individual, dependiendo de la distinta capacidad de cada sujeto de adaptación a las exigencias del entorno social y laboral, en función de múltiples factores personales (NTP 534 y 349) ${ }^{(17-18)}$. Ante condiciones similares, algunos individuos desarrollan niveles de estrés patológico y otros no, debido a diferentes características o condicionamientos personales (carencia o inadecuada utilización de conductas de afrontamiento frente a las demandas del medio y desarrollo de reacciones fisiológicas y/o emocionales inadecuadas). 
Figura 2. Salud mental de los trabajadores y prevención de riesgos laborales

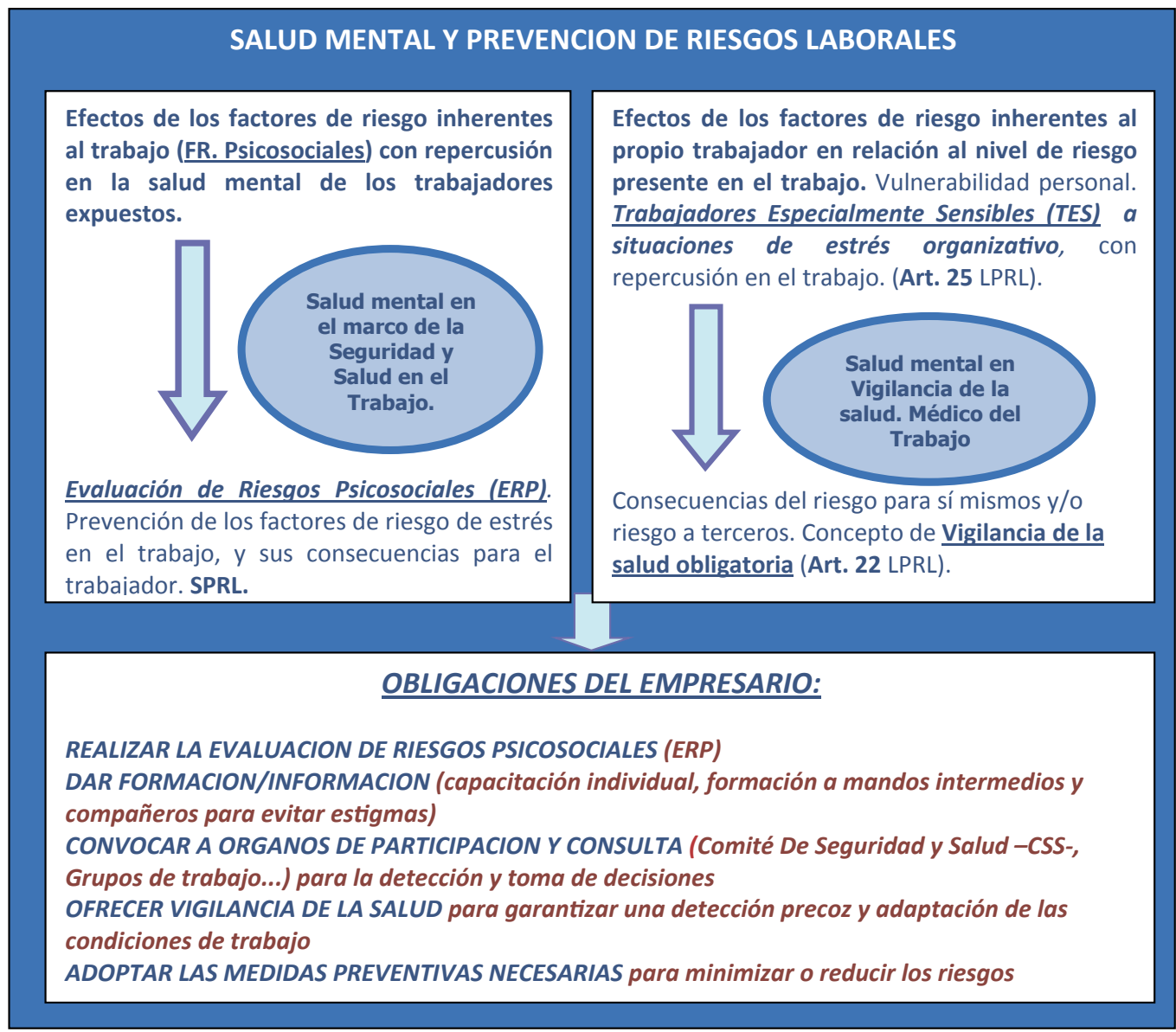

\section{B) Influencia del estado de salud mental en el trabajo}

Cuando se altera la funcionalidad del individuo afectado por un trastorno mental para desarrollar su actividad laboral, necesita un periodo de incapacidad (baja por enfermedad) hasta su recuperación total o parcial. Cuando esta funcionalidad no se ve apenas alterada, permitiéndole afrontar los requerimientos laborales habituales, aunque con mayor riesgo personal de empeorar su estado de salud mental, hablamos de Trabajadores Especialmente Sensibles (TES) frente al riesgo de estrés organizativo, en los términos que desarrolla el art. 25 de la LPRL:

"El empresario garantizará de manera específica la protección de los trabajadores que, por sus propias características personales o estado biológico conocido, incluidos aquellos que tengan reconocida la situación de discapacidad física, psíquica o sensorial, sean especialmente sensibles a los riesgos derivados del trabajo. A tal fin, deberá tener en cuenta dichos aspectos en las evaluaciones de los riesgos y, en función de éstas, adoptará las medidas preventivas y de protección necesarias.

Los trabajadores no serán empleados en aquellos puestos de trabajo en los que, a causa de sus características personales, estado biológico o por su discapacidad física, psíquica o sensorial debidamente reconocida, puedan ellos, los demás trabajadores $u$ otras personas relacionadas con la empresa ponerse en situación de peligro o, en general, cuando se encuentren manifiestamente en estados o situaciones transitorias que no respondan a las exigencias psicofísicas de los respectivos puestos de trabajo". 
La Guía de buenas prácticas para la prevención y manejo de problemas de salud mental en trabajadores del ámbito sanitario(7) (ISCIII, 2012) propone, entre otras, una herramienta de trabajo novedosa para la detección de TES frente al riesgo de estrés organizativo. Propone la valoración de tres Indicadores de riesgo, que complementarían a la entrevista personal al trabajador (en el contexto de la Historia clínico-laboral):

1. Escala de salud general (modificada de Cuestionario de Goldberg GHQ-12) autocumplimentada por el trabajador, con puntuación igual o mayor a 3 (Figura 3).

2. Presencia de antecedente personal de trastorno mental y/o consumo de abuso.

3. Presencia de condiciones psicosociales adversas (personales, familiares, laborales, o una combinación de ellas).

Cuando se obtenga una puntuación igual o superior a 3 en la Escala de salud general, combinado con la presencia de algún otro indicador de riesgo personal, se considerará TES frente al riesgo psicosocial o riesgo de estrés, y se procederá solicitar una valoración especializada (psicopatológica) del trabajador por profesionales de la salud mental antes de valorar la aptitud según el riesgo especifico de su puesto de trabajo (riesgo a sí mismo y riesgo a terceros).

Figura 3. Ficha autocumplimentada para valoración de TES (ISCIII, 2012)

Datos a rellenar por el TRABAJADOR:

\begin{tabular}{|c|c|c|c|c|}
\hline \multicolumn{5}{|c|}{$\begin{array}{l}\text { 1.Escala de Salud (modificado de GHQ-12): } \\
\text { tache con "X" el número elegido, con el que vd. se identifique más, de } 0 \text { a } 3\end{array}$} \\
\hline (items positivos) DURANTE EL ULTIMO MES... & $\begin{array}{l}\text { Más que lo } \\
\text { habitual (0) }\end{array}$ & $\begin{array}{c}\text { Tgual que } \\
\text { habitualmente (1) }\end{array}$ & $\begin{array}{l}\text { Mennos da lo } \\
\text { habitual(2) }\end{array}$ & $\begin{array}{l}\text { Mucho manns } \\
\text { de habitual (3) }\end{array}$ \\
\hline \multicolumn{5}{|l|}{ ¿ha podido concentrarse bien en lo que hace? } \\
\hline \multicolumn{5}{|l|}{ ¿ha sentido que estajugando un papelútlen la vida? } \\
\hline \multicolumn{5}{|l|}{ ¿se ha sentdo capaz de tomar decisiones? } \\
\hline \multicolumn{5}{|l|}{ ¿ha sido capaz de disfrutar sus actividades normales diarias? } \\
\hline \multicolumn{5}{|l|}{ ¿ha sido capaz de hacer frente a sus problemas? } \\
\hline \multicolumn{5}{|l|}{ ¿se siente razonablemerte feliz con todas circunstancias? } \\
\hline (items negativos) DURANTE EL ULTIMO MES... & $\begin{array}{c}\text { No en } \\
\text { absoluto(0) }\end{array}$ & $\begin{array}{l}\text { No más que lo } \\
\text { habitual(1) }\end{array}$ & $\begin{array}{l}\text { más de lo } \\
\text { habitual(2) }\end{array}$ & $\begin{array}{c}\text { Mucho más } \\
\text { de habilual(3) }\end{array}$ \\
\hline \multicolumn{5}{|l|}{ ¿sus preocupaciones le hacen perder mucho sueño? } \\
\hline \multicolumn{5}{|l|}{ ¿se ha sentido constantemente agobiado y en tensión? } \\
\hline \multicolumn{5}{|l|}{ ¿ha sentido que no puede superar sus diffcultades? } \\
\hline \multicolumn{5}{|l|}{ ¿se ha sentido deprimido o infeliz? } \\
\hline \multicolumn{5}{|l|}{ ¿ha perdido confiarza en si mismo? } \\
\hline ¿ha pensado que vd es una persona que no sirve para nac & & & & \\
\hline
\end{tabular}

2.Sintomas que ha presentado durante el último mes: (codificar como X si la respuesta es afirmativa)

Sentimiento de irritabilidad, nerviosismo o tensión emocional

Dificultad para relajarse o conciliar el sueño

Alguno/s de estos: cefaleas, cervicalgias, tensión muscular, temblores, sudores, hormigueos, diarrea Dificultad en la concentración y atención

Perdida reciente de apetito y/o pérdida reciente de peso

Sensación de falta de energia reciente, cansancio desde por las mañanas

Sentimientos depresivos, tristeza, apatía

Ausencia de sintomas, sensación de bienestar físico y psíquico

Otros (especificar):

3.Situación sociofamiliar: (codificar como $X$ si la respuesta es afirmativa)

Importante carga familiar (hijos/padres a su cargo) y/o importante carga económica

Dificultad en las relaciones familiares/amistades (poca dedicación por falta de tiempo, etc)

Dificil adaptación al entorno social, relaciones interpersonales en genera

Declaro que toda la información aportada es veraz.

Nombre y Apellidos del Trabajador:

Fdo.:

En a de de 2 
Corrección Cuestionario modificado de Goldberg-12 (GHQ-12):

Contabilizar las puntuaciones 0 y 1 como 0

Contabilizar las puntuaciones 2 y 3 como 1

(Puntuación máxima posible $=12$ ). Punto de Corte $=3$.

Indicadores de TES para solicitar la valoración psiquiátrica del trabajador:

Cuestionario modificado de Goldberg con puntuación $\geq 3$.

Además señalar la existencia de: antecedente de trastorno mental previo (+) y/o Consumo de sustancias de abuso (+) y/o Condiciones psicosociales de riesgo (+).

En general, serían “TES para el riesgo de estrés organizativo” los trabajadores con mala respuesta al estrés (cuadros de ansiedad importantes, severas somatizaciones, etc.), con antecedentes personales de trastornos psicológicos, o de conductas adictivas previas, con historia familiar de adicciones, o con alto riesgo socio-familiar (divorcios traumáticos, presión social, etc.). A título orientativo, en la Guía del ISCIII se detallan algunas situaciones revisadas:

- Acreditación de reconocimiento de grado de minusvalía psíquica por trastorno mental, incluyendo la discapacidad intelectual (Guía para la gestión de la Prevención de Riesgos laborales de trabajadores con discapacidad intelectual, 2009) ${ }^{(19)}$. Los trabajadores se incorporan a la organización con un porcentaje reconocido de minusvalía psíquica. Debe tenerse en cuenta el déficit que presentan, para establecer adaptaciones laborales específicas (existen organizaciones adaptadas a las características de trabajadores discapacitados Hilarión y Koatz, 2012) ${ }^{(20)}$.

- Los antecedentes de trastorno mental previo o actual con repercusión funcional de diverso grado (como la esquizofrenia, el trastorno obsesivo compulsivo o el trastorno bipolar), pueden ser compatibles con la actividad profesional (Mingote y col, 2007-2011) ${ }^{(21-24)}$, dependiendo de la evolución y del tratamiento con psicofármacos (aunque el consumo de ansiolíticos o antidepresivos, en sí mismo, no es criterio de gravedad). Cuando la afectación de la funcionalidad repercute en la actividad laboral (dificultad para solventar problemas, reacciones emocionales inadecuadas en los enfrentamientos, o bien por una dificultad importante o incapacidad para la toma de decisiones) se revisará la aptitud laboral.

- Características de personalidad o rasgos de carácter "facilitadores" de percepción de distress y agotamiento profesional (perfeccionistas, obsesivos, inseguros, con baja autoestima, etc.). Cuando los rasgos de personalidad son inflexibles y maladaptativos, y suponen un deterioro significativo, pueden diagnosticarse de trastorno de la personalidad. Han de tenerse en cuenta los trastornos esquizotípico, paranoides o límites, para tareas de especial responsabilidad, y los trastornos paranoides, cuando el delirio tiene una clara relación con el ámbito laboral (Gálvez y col, 2010)(25).

- Conductas adictivas, aún referidas al tiempo de ocio, con repercusión funcional (Otero, 2008, 2012) $)^{(26-27)}$.

- Alteración en la percepción del propio estado de salud: ausencia de conciencia de enfermedad en enfermedades crónicas que impliquen deterioro de la capacidad funcional, trastornos somatomorfos (presencia no intencionada de síntomas de malestar clínico o deterioro funcional significativo sugerentes de enfermedad somática, que no pueden explicarse completamente, y que su origen se encuentra en un incorrecto manejo por parte del paciente de un estresor psíquico, debido a unos rasgos anómalos en su personalidad) y alteraciones somáticas con excesiva reactividad al estrés cotidiano (insomnio crónico, etc.). 
- Vivencia reciente de experiencias estresantes específicas graves con repercusión en la salud mental (acontecimientos traumáticos, muerte de un familiar próximo, separación de la pareja, situaciones económicas desfavorables, etc.).

En el caso de detectar situaciones de riesgo, se podrán proponer adaptaciones del puesto (p. ej. reincorporación laboral progresiva a tareas de responsabilidad, evitar turnos cambiantes, etc.) e intervenciones individuales sobre el trabajador (seguimiento periódico personalizado, información sobre los riesgos a los que está expuesto y formación en estrategias de afrontamiento para mejorar su autoestima, responsabilidad en toma de decisiones, reducción de ansiedad, etc.). Se debe ofrecer al trabajador la posibilidad de derivación a opciones terapéuticas por profesionales de la salud mental y/o a programas específicos de la red asistencial.

\section{Efectos de la pérdida de salud mental, funcionalidad y competencias del individuo enfermo}

En realidad, no es el diagnóstico de un trastorno mental, en sí mismo, lo que determina la duración del periodo de IT y la reincorporación laboral del trabajador afectado, sino el grado de competencia o suficiencia para realizar la actividad laboral que venía desarrollando, el funcionamiento global del individuo, así como la capacidad de la organización para integrar al trabajador en un entorno saludable de trabajo de adecuada compatibilidad.

Ante la reincorporación laboral de un trabajador tras un periodo de ausencia laboral prolongado, por un motivo de alteración de su salud mental, se valorarán ${ }^{(7)}$ :

1. Anamnesis e bistoria clinicolaboral: contemplando antecedentes psiquiátricos y laborales previos (exposición a factores de riesgo psicosocial).

a) Antecedentes psiquiátricos: valorar la gravedad diagnóstica (psicosis, depresión mayor, trastorno neurótico severo, crisis adaptativa severa, alcoholismo y adicciones severas, trastornos alimentarios graves), otros signos de gravedad (pensamientos de auto/heterolesión, cuadros de delirios o alucinaciones), comorbilidad, evolución aguda o crónica del proceso.

b) Antecedentes orgánicos: patología crónica asociada, cuadros neurológicos, trastornos del sueño (SAOS, etc.) y cualquier proceso que pueda agravar su estado actual. Accidentes domésticos y/o de tráfico previos, etc.

c) Antecedentes laborales: exposición mantenida a elevados niveles de riesgo psicosocial y desarrollo de síntomas o procesos de burnout, situaciones detectadas de violencia interna o externa en la organización, condición previa de TES al riesgo organizativo de estrés, antecedentes de reclamaciones de usuarios y/o quejas reiteradas de compañeros y superiores, accidentes de trabajo previos (analizar el mecanismo de producción de los mismos).

2. Estado actual: valorando el grado de limitación del trabajador afectado de un trastorno mental. Valorar a través de sencillas preguntas (Figura 4) la limitación y, además:

a) Conciencia de enfermedad.

b) Tratamiento psiquiátrico y grado de adherencia al mismo. Tipo y dosis de psicofármacos. Efectos.

c) Disponibilidad de apoyo socio-familiar.

d) En caso de toxicodependencias: posibilidad de establecer un compromiso terapéutico y de controlar su eficacia y continuidad y para la prevención de recaídas.

3. Valoración del nivel de riesgo del puesto de trabajo actual:

a) Del riego psicosocial: exposición mantenida a elevados niveles de riesgo psicosocial, con presencia de estresores ambientales (p.ej.: ruido, dificultad 
para la adecuada regulación de la temperatura), organizativos (p.ej.: conflictos con compañeros, turnos cambiantes, prolongación de jornadas), etcétera.

b) Del riesgo para sí mismo y para terceros:

i) Autoconciencia de riesgo. Se debe tener en cuenta tanto la conciencia o la ausencia de conciencia de riesgo por el propio trabajador.

ii) Evaluar la magnitud del riesgo que existe en el desempeño de su tarea para sí mismo y para los otros (P. ej.: manejo de maquinaria peligrosa, trabajo en altura, cortes y punciones, conducción de vehículo y/o transportes de pasajeros, tareas que precisan un alto nivel de atención y toma rápida de decisiones como la práctica quirúrgica, etc.).

4. Capacidad de la Organización para asumir la propuesta de adaptación o cambio de puesto de trabajo (cambios de turno, reducción de jornada, evitación de tareas de mayor complejidad, asumir responsabilidades de forma progresiva y tutorizada, etcétera).

Figura 4. Preguntas para aproximarse a la limitación del trabajador afectado de un trastorno mental. Tomado de la "Guía de valoración de incapacidad laboral para médicos de atención primaria"

\section{PREGUNTARNOS Y PREGUNTAR AL TRABAJADOR:}

- ¿Es capaz de realizar su trabajo dentro de su jornada laboral habitual (horario, ritmo, desplazamiento...)?

- ¿Es capaz de realizar su trabajo dentro del entorno material y humano habituales (lugar de trabajo, compañeros, usuarios...)?

- ¿Es capaz de realizar su tarea sin cometer errores que puedan repercutir en su seguridad o la de otros (tomar decisiones rápidamente, prestar atención mantenida, etc.)?

La aptitud laboral es la valoración de la relación que se produce entre:

1. las capacidades psicofísicas del trabajador para realizar una actividad laboral específica (valorando el grado de limitación que el trabajador afectado de un trastorno psíquico y/o físico pueda padecer) $\mathrm{y}$

2. las demandas que le impone su puesto de trabajo (Valorando el nivel de riesgo del puesto de trabajo actual según las características de las tareas, entorno y equipos de trabajo, características de la organización a la que pertenece, etc.).

Para ello, el médico del trabajo podrá realizar las medidas de vigilancia de la salud que estime necesarias y proporcionales al riego (art. 22.1 LPRL), con respeto a la intimidad y confidencialidad del estado de salud del trabajador (art. 22.2) y cuyos resultados no podrán ser usados con fines discriminatorios ni en perjuicio del trabajador (art. 22.4 LPRL). La exploración de la salud mental del trabajador debe estar integrada en la propia actividad de la vigilancia de la salud (valoración de la aptitud psicofísica del trabajador), con los medios que el médico del trabajo tenga a su alcance.

En este sentido, la Guía de buenas prácticas (ISCIII, 2012)(7), propone una metodología para determinar la aptitud para el puesto de trabajo, teniendo en cuenta las competencias de los trabajadores (valorando el estado clínico y funcional del trabajador afectado), así como la magnitud del riesgo según las tareas que realiza, aunque, en última instancia la valoración es siempre individual y tendrá en cuenta la capacidad de la organización de asumir la adaptación específica en cada caso, y la existencia planes de prevención en la empresa, generales (Plan de Ayuda al Empleado) y específicos (Plan de prevención de drogodependencias, de burnout, de violencia interna, etc.). 


\section{Algunas consideraciones específicas en materia de seguridad individual y/o colectiva}

Aunque el riesgo es una variable dependiente del contexto, pueden darse conductas de riesgo en algunos trabajadores asociadas a una dificultad del control de impulsos o a un déficit atencional en adultos con un Trastorno por Déficit de Atención e Hiperactividad $\left(\right.$ TDAH) ${ }^{(28)}$ (Delisle y Braun, 2011), o por hipersomnolencia diurna (en Síndrome de Apnea Obstructiva del Sueño, SAOS), o por un déficit cognitivo asociado a fases incipientes de procesos neurológicos (fases iniciales de demencia, etc.).

Todos estos procesos podrían tener serias consecuencias si afectasen a trabajadores con importante responsabilidad sobre terceros (conductores de vehículos, sobre todo los que se dedican al transporte de pasajeros, manejo de maquinaria o sustancias peligrosas, etc.) y pueden agravarse en el trabajo a turnos/nocturno.

La valoración de la aptitud, la propuesta de adopción de medidas adaptativas en el puesto de trabajo, así como el tratamiento del proceso y el seguimiento de su evolución, se beneficiarían de la posibilidad de realizar estudios neuropsicológicos a dichos trabajadores (capacidad cognitiva), entre otras pruebas diagnósticas (pruebas de imagen, como TAC o RMN, p. ej.).

\section{Gestión sanitaria de la pérdida de la salud mental en el ámbito laboral}

En el ámbito de la gestión sanitaria (Fig. 5), la coordinación de recursos y la comunicación efectiva entre el médico del trabajo del SPRL, el médico de familia, el profesional/especialista de salud mental y el médico evaluador del Equipo de Valoración de Incapacidades (EVI) del Instituto Nacional de la Seguridad Social (INSS), resultan imprescindibles para garantizar la reincorporación laboral del trabajador con un trastorno de salud mental en las mejores condiciones de seguridad y salud (Mingote y Núñez, 2011) ${ }^{(29)}$

- La prestación sanitaria es competencia del especialista de salud mental que trata al paciente, considerando durante ese proceso la posibilidad de mantener la actividad laboral (paciente en activo) o la conveniencia de evitar la actividad laboral, en relación a la situación de riesgo-beneficio para su paciente.

- La valoración de la aptitud laboral para el puesto de trabajo específico es competencia del médico especialista en medicina del trabajo, cuando el paciente se encuentra en situación activa laboral, para que el empresario pueda garantizar un entorno seguro y saludable (art. 22.4 LPRL). Cuando un trabajador se reincorpora a su puesto de trabajo tras una ausencia prolongada por motivos de salud mental, corresponde al médico del trabajo evaluar la aptitud laboral (art. 37.3.a) determinando quienes serían TES frente al riesgo de estrés organizativo (art. 22 y 25 de la LPRL) y proponiendo adaptación o cambio del puesto de trabajo durante un periodo de seguimiento personalizado. El médico del trabajo debería tener información objetiva del grado de afectación del trastorno mental (procesos psicológicos afectados, medicación que está tomando al alta, etc.), que habitualmente le pedirá al propio trabajador (informes del especialista de salud mental).

- La gestión sanitaria de la prestación de la Incapacidad Temporal (IT) es competencia del médico de familia de Atención Primaria de la Red Sanitaria Pública, o de su Mutua de Accidentes de Trabajo y Enfermedad Profesional de la Seguridad Social (MATEPSS), en caso de tener concierto de gestión de "Enfermedad Común (EC)”. Es el médico de familia quien decide la duración de la IT, evaluando el estado psicofísico del paciente y los informes del especialista de salud mental que le trata. Para ello dispone, además de su experiencia profesional, de diversas guías de consulta (p.ej. Manual de tiempos óptimos de incapacidad temporal ${ }^{(30)}$ (2014) elaborada por el INSS, que detalla un listado tiempos de baja estimados para más de 150 procesos diagnósticos de trastorno mental, o la Guía de valoración de incapacidad laboral para médicos de atención primaria $^{(31)}$ (2010), 
que establece una relación entre los grados de limitación de las patologías psiquiátricas y la necesidad de IT). Sin embargo, el médico de familia recibe una información subjetiva (procedente de la propia percepción del paciente) sobre el entorno de trabajo. Lo ideal sería que, antes de emitir el alta, el médico de familia pudiese recabar, al médico del trabajo del SPRL (directamente o a través de la Inspección Médica), información objetiva de las condiciones del puesto de trabajo del paciente común.

- La gestión de la prestación por Incapacidad Permanente (IP) es competencia del INSS. Los médicos evaluadores del EVI, a partir de un informe médico de propuesta de incapacidad (procesos o patologías graves, habitualmente crónicas y/o progresivas, o procesos que presentan una alteración cognitiva asociada y una alteración de la percepción de la realidad y solo remiten parcialmente), valoran al enfermo y determinan la existencia o no de incapacidad laboral permanente y la gravedad de la misma. El médico evaluador del EVI dispone, además de su experiencia profesional, de diversas guías de consulta en cuanto a los trastornos mentales, como el Manual de Evaluación de Requerimientos para la Valoración de la Capacidad Laboral (MERCAL-2008) ${ }^{(32)}$, muy buena herramienta para valorar los requerimientos psicológicos asignados a las diferentes categorías profesionales, aproximándose al concepto de competencia profesional, sin embargo el informe médico del médico del trabajo del SPRL al INSS, es la herramienta más específica para cada caso concreto. En este sentido, en 2011 se consensuó un sistema de colaboración entre los SPRL del Servicio Madrileño de Salud (SERMAS) y el INSS.

Figura 5. Coordinación de recursos sanitarios en la gestión de la salud mental de los trabajadores

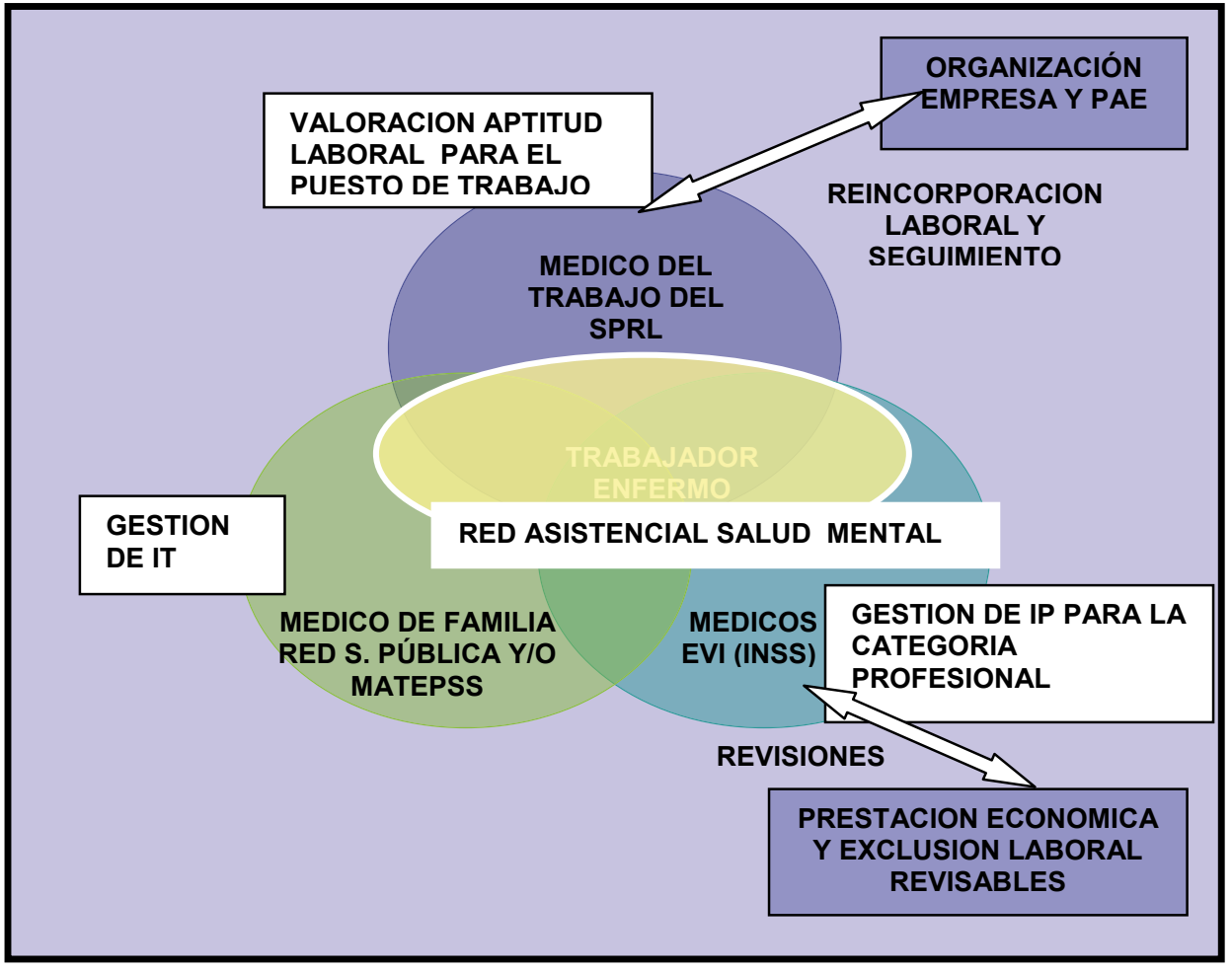

\section{Posibles barreras a la reincorporación}

La reincorporación laboral del trabajador con problemas de salud mental se puede complicar con barreras asociadas al propio contexto (estigma, clima laboral, interactuación con otros trabajadores y/o usuarios...), al propio trabajador (alteraciones afectivas, 
cognitivas, emocionales...), y a la tarea que venía realizando [con elevadas exigencias cognitivas y/o sensoriales, NTP 659(33)].

Los Programas de Ayuda al Empleado (PAE) tienen su origen en USA, en los años 80, y desde entonces se han ido consolidando en Europa y tienen una consideración muy positiva en las grandes empresas [NTP $\left.780^{(12)}\right]$ porque:

- Contribuyen a mejorar la salud mental entre los trabajadores (mejorando el rendimiento individual o de grupo, contribuyendo a disminuir el absentismo, y en definitiva, a la mejora de la productividad) y

- se puede utilizar como una herramienta de gestión para identificar y abordar situaciones conflictivas).

El Programa de Fomento del Empleo y la. Solidaridad Social de la Unión Europea PROGRESS (2007-2013) ${ }^{(34)}$, ha financiado estudios y publicaciones de documentos en este sentido [Salud Mental y Empleo. Guía para empleadores ${ }^{(35)}$ ] para superar las barreas que pueda encontrarse el trabajador que se reincorpora tras una baja por problema de salud mental. Se propone un RETORNO AL TRABAJO planificado con la persona afectada y su supervisor o responsable de área la reincorporación al trabajo, recomendando utilizar durante este proceso el asesoramiento del Servicio de Salud Laboral de la empresa, si se dispone de él, y estableciendo la información sobre su enfermedad que el trabajador quiere que se conozca por sus mandos superiores y compañeros (Fig. 6):

Figura. 6. Objetivos del Retorno al Trabajo. Adaptado de "Retorn al treball després d'una malaltia mental" (Solé D.) (36)

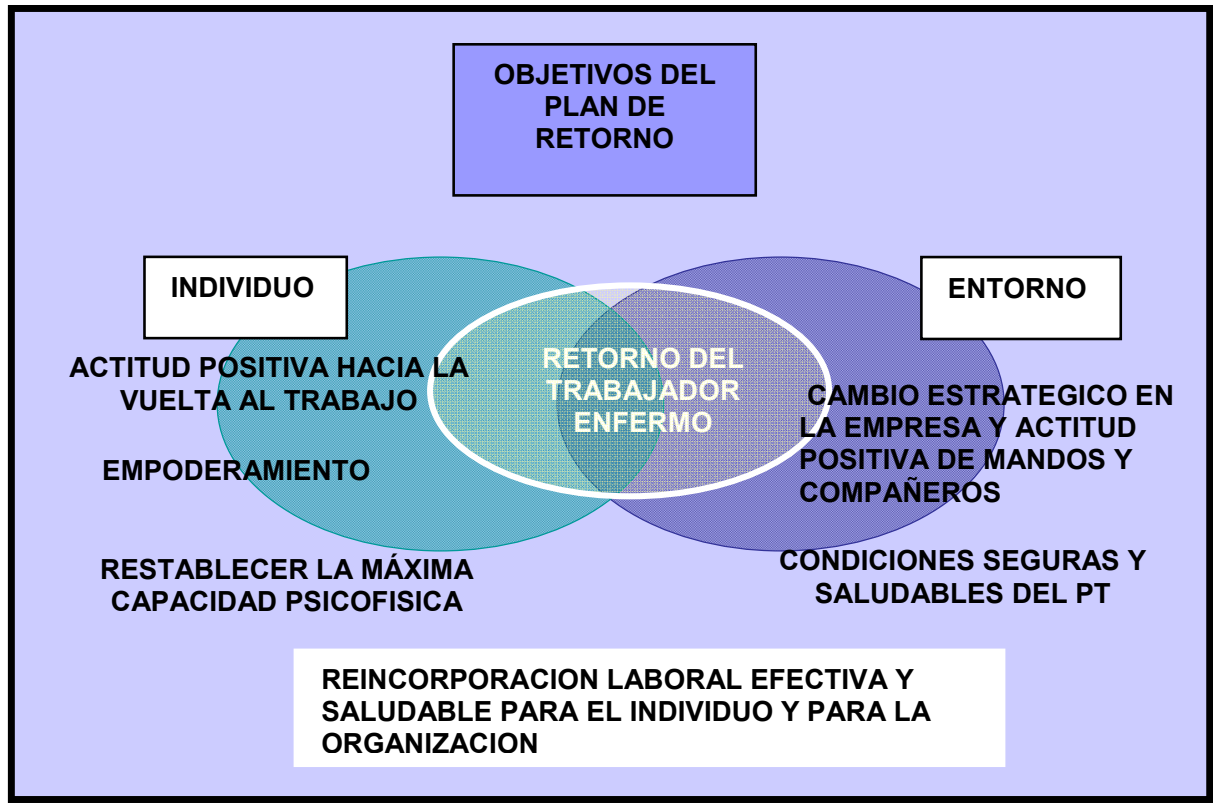

Ejemplos de Planes de Ayuda a empleados y reincorporación laboral en nuestro medio, en el sector sanitario, son el Programa Retorno para enfermería y el Programa PAIME para médicos, puestos en marcha por la mayoría de los Colegios Profesionales. También en otras empresas, la mayoría del sector servicios (p.ej.: Programa Incorpora de "la Caixa"), o incluso en programas diseñados para la reinserción laboral a diferentes empresas desde el punto de vista de la terapia ocupacional (Sánchez, 2006) ${ }^{(37)}$.

\section{BIBLIOGRAFÍA}

1. RD 39/1997, de 17 de enero, por el que se aprueba el Reglamento de los Servicios de Prevención (BOE 27,31-En-1997). http://www.boe.es. 
2. Ley 31/1995, de 8 de noviembre, Ley de Prevención de Riesgos Laborales (BOE 269, 10-Nov-1995). http:// www.boe.es.

3. NTP 780. El programa de ayuda al empleado (EAP): intervención individual en la prevención de riesgos psicosociales. INSHT. Disponible en: http://www.insht.es/InshtWeb/Contenidos/Documentacion/ FichasTecnicas/NTP/Ficheros/751a785/780\%20.pdf.

4. Crawford, J. O., George, P., Graveling, R. A., Cowie, H., Dixon, K. Good Work, Good Health. IOM (Institute of Occupational Medicine de Edimburgo). Informe de Investigación 603-00944. Jun, 2010. http://www.gwgh.eu/.

5. European Survey of New and Emerging Risks, ESENER (OSHA-2012). Disponible en: https://osha.europa eu/es/about/calls/eu-osha-pru-2012-p-03-second-european-survey-of-enterprises-on-new-and-emergingrisks-esener-2.

6. The Good Practice Guidelines on how to improve the mental well-being of workers in the telecommunications sector (2010). Good Work, Good Health site: http://www.gwgh.eu/.

7. Gálvez Herrer, M., Mingote Adán, C., Núñez López, C., Otero Dorrego, C. Coordinación: Otero Dorrego, C. "Guía de buenas prácticas para la prevención y manejo de problemas de salud mental en trabajadores del ámbito sanitario”. Escuela Nacional de Medicina del Trabajo, Instituto de Salud Carlos III - Ministerio de Ciencia e Innovación. Abril 2012. Disponible en: http://publicaciones.isciii.es/unit.jsp?unitId=enmt.

8. Trabajemos contra el estrés. Prevención de riesgos psicosociales y estrés laboral en la práctica. Agencia Europea para la Seguridad y la Salud en el Trabajo, 2003. Dirección de Internet: http://europa.eu.int.

9. Fundación Europea para la Mejora de las Condiciones de Vida y de Trabajo. Cuarta encuesta europea sobre las condiciones de trabajo (2007), Oficina de Publicaciones Oficiales de las Comunidades Europeas, Luxemburgo, 2007 http://www.eurofound.europa.eu/ewco/surveys/EWCS2005/index.htm.

10. Gil-Monte, P. R. Algunas razones para considerar los riesgos psicosociales En el trabajo y sus consecuencias en la salud pública. Rev Esp Salud Pública 2009; 83: 169-173.

11. VII Encuesta Nacional de Condiciones de Trabajo. INSHT, 2011. http://www.oect.es/InshtWeb/ Contenidos/Documentacion/FICHAS\%20DE\%20PUBLICACIONES/EN\%20CATALOGO/OBSERVATORIO/ Informe\%20(VII\%20ENCT).pdf.

12. NTP 702: El proceso de evaluación de los factores psicosociales. INSHT. Disponible en: http://www.insht. es/InshtWeb/Contenidos/Documentacion/FichasTecnicas/NTP/Ficheros/701a750/ntp_702.pdf.

13. NTP 704: Síndrome de estar quemado por el trabajo o "burnout" (I): definición y proceso de generación. INSHT. Disponible en: http://www.insht.es/InshtWeb/Contenidos/Documentacion/FichasTecnicas/NTP/ Ficheros/701a750/ntp_704.pdf.

14. NTP 705: Síndrome de estar quemado por el trabajo o "burnout" (II): consecuencias, evaluación y prevención. INSHT. Disponible en: http://www.insht.es/InshtWeb/Contenidos/Documentacion/ FichasTecnicas/NTP/Ficheros/701a750/ntp_705.pdf

15. NTP 732: Síndrome de estar quemado por el trabajo "Burnout" (III): Instrumento de medición. INSHT. Disponible en: http://www.insht.es/InshtWeb/Contenidos/Documentacion/FichasTecnicas/NTP/Ficheros/701a750/ntp_732. pdf.

16. NTP 854. Acoso psicológico en el trabajo: definición. INSHT. Disponible en: http://www.insht.es/ InshtWeb/Contenidos/Documentacion/FichasTecnicas/NTP/Ficheros/821a921/854\%20web.pdf.

17. NTP 534: Carga mental de trabajo: factores. INSHT. Disponible en: http://www.insht.es/InshtWeb/ Contenidos/Documentacion/FichasTecnicas/NTP/Ficheros/501a600/ntp_534.pdf.

18. NTP 349: Prevención del estrés: intervención sobre el individuo. INSHT. Disponible en: http://www. insht.es/InshtWeb/Contenidos/Documentacion/FichasTecnicas/NTP/Ficheros/301a400/ntp_349.pdf.

19. Guía para la gestión de la Prevención de Riesgos laborales de trabajadores con discapacidad intelectual. Foment del Treball. Fundacion Prevent. 2009. Disponible en: http://www.fundacionprevent.com/ Actividades/guias_foment/PRL_discap_intelectual.pdf

20. Hilarión, P., Koatz, D. Guía para la integración laboral de personas con trastorno mental. Coordinación: Suñol R, Hilarión P. Programa Incorpora de"la Caixa”. Obra social La Caixa (2012). Disponible en: http:// www.feafes.org/publicaciones/Guiaparaintegracionlaboralenfermedadmental.pdf.

21. Mingote, J. C., del Pino, P., Sánchez, R., Gálvez, M. y Gutiérrez, M. D. El trabajador con problemas de salud mental. Pautas generales de detección, prevención e intervención. Med Segur Trab (Internet) 2011; 57. Suplemento 1: 1-262. Disponible en: http://scielo.isciii.es/pdf/mesetra/v57s1/actualizacion10.pdf.

22. Mingote Adán, J. C., Pino Cuadrado, P., Gálvez Herrer, M., Gutiérrez García, M. D., Sánchez Alaejos, R. Utilidad preventiva del constructo "trastorno mental grave" en el ámbito sociosanitario. Med. segur. trab. [revista en la Internet]. 2010 Dic [citado 2013 Sep 22]; 56(221): 306-322. Disponible en: http://scielo.isciii.es/scielo. php?script=sci_arttext\&pid=S0465-546X2010000400006\&lng=es. doi: $10.4321 /$ S0465-546X201000040000 
23. Mingote Adán, J. C., Pino Cuadrado, P., Huidobro, A., Gutiérrez García, D., Miguel Peciña, I., Gálvez Herrer, M. El paciente que padece un trastorno psicótico en el trabajo: diagnóstico y tratamiento. Med. segur. trab. [revista en la Internet]. 2007 Sep [citado 2013 Sep 28] ; 53(208): 29-51. http://scielo.isciii. es/scielo.php?script=sci_arttext\&pid=S0465-546X2007000300005\&lng=es; http://dx.doi.org/10.4321/ S0465-546X2007000300005.

24. Mingote Adán, J. C., Gálvez Herrer, M., Pino Cuadrado, P., Gutiérrez García, M. D. El paciente que padece un trastorno depresivo en el trabajo. Med. segur. trab. [revista en la Internet]. 2009 Mar [citado 2013 Sep 28] ; 55(214): 41-63. Disponible en: http://scielo.isciii.es/scielo.php?script=sci_arttext\&pid=S0465546X2009000100004\&lng=es. http://dx.doi.org/10.4321/S0465-546X2009000100004.

25. Gálvez Herrer, M., Mingote Adán, J. C., Moreno Jiménez, B. El paciente que padece un trastorno de personalidad en el trabajo. Med. segur. trab. [revista en la Internet]. 2010 Sep [citado 2013 Sep 28] ; 56(220): 226-247. Disponible en: http://scielo.isciii.es/scielo.php?script=sci_arttext\&pid=S0465546X2010000300005\&lng=es. http://dx.doi.org/10.4321/S0465-546X2010000300005.

26. Otero Dorrego, C., Huerta Camarero, C., Duro Perales, N. Drogodependencias en personal sanitario, una visión desde la medicina del trabajo (II): Procedimiento de reincorporación laboral. Med. segur. trab. [revista en la Internet]. 2008 Sep [citado 2013 Sep 28] ; 54(212): 7-19. Disponible en: http://scielo.isciii. es/scielo.php?script=sci_arttext\&pid=S0465-546X2008000300002\&lng=es. $\quad$ http://dx.doi.org/10.4321/ S0465-546X2008000300002.

27. Otero Dorrego, C. Drogodependencias en el lugar de trabajo: Pautas generales de intervención desde la medicina del trabajo. Med. segur. trab. [revista en la Internet]. [citado 2013 Sep 28]. Disponible en: http://scielo.isciii.es/scielo.php?script=sci_arttext\&pid=S0465-546X2011000500010\&lng=es. $\quad \mathrm{http}: / / \mathrm{dx}$. doi.org/10.4321/S0465-546X2011000500010.

28. Delisle J, Braun, C. M. J. Un contexto para la normalización de la impulsividad en el trabajo para los adultos con Déficit de Atención e Hiperactividad. Archives of clinical Neuropsychology. 2011 Jun. Disponible en: http://www.psiquiatria.com/articulos/hiperactividad/52337/.

29. Mingote, J. C. y Núñez, C. Importancia de la consideración de la salud mental en la gestión de la salud laboral: una responsabilidad compartida. Med Segur Trab (Internet) 2011; 57. Suplemento 1: 1-262. Disponible en: http://scielo.isciii.es/pdf/mesetra/v57s1/actualizacion13.pdf.

30. Manual de tiempos óptimos de incapacidad temporal (3. ${ }^{a}$ ed.). INSS. 2014.Disponible en: http://www. seg-social.es/Internet 1 /LaSeguridadSocial/Publicaciones/Publicacionesporcon 28156/ Informacionsobrepen47075/Incapacidadtemporal/index.htm\#documentoPDF.

31. Guía de valoración de incapacidad laboral para médicos de atención primaria. Monografías ISCIII. 2010. Disponible en: http://www.tt.mtin.es/periodico/seguridadsocial/201101/GUIA_DE_VALORACION_DE_ INCAPACIDAD_LABORAL_PARA_AP.pdf.

32. Manual de evaluación de requerimientos para la valoración de la capacidad laboral (MERCAL-2008). Disponible en: http://www.cop.es/delegaci/andocci/files/noticias/1817/protocolo\%20manual\%20 requerimientos\%20valoracion\%20capacidad\%20laboral\%2030-12-08.pdf.

33. NTP 659: Carga mental de trabajo: diseño de tareas. Disponible en: http://www.insht.es/InshtWeb/ Contenidos/Documentacion/FichasTecnicas/NTP/Ficheros/601a700/ntp_659.pdf.

34. European Network for Workplace Health Promotion. Employee Assistance Programme. Disponible en: https://www.google.es/\#psj=1\&q=ENWHP+Toolbox

35. Salud Mental y Empleo. Guía para empleadores. Disponible en: http://www.insht.es/PromocionSalud/ Contenidos/Promocion\%20Salud\%20Trabajo/Ambitos/ficheros/SaludMental_Empleo_GuiaEmpleadores. pdf.

36. Solé, D. Retorn al treball després d'una malaltia mental. Eines de canvi: Promoció del benestar física, psíquic i social de les persones i organitzacions. INSHT. Disponible en: http://www.incorporasaludmental. org/images/doc/D_CAS_EMP_EINA_RETOR_0330_Ret_treba_desp_malal_mental.pdf.

37. Sánchez, O. Rehabilitación Laboral de Personas con Enfermedad Mental 2006. Disponible en: http:// www.psicosocialart.es/documentacion/word/seminariorehlab.pdf. 